\begin{tabular}{|l|c|c|c|c|c|}
\hline Cuadernos I. Geográfica & 16 & $1-2$ & $85-98$ & Logroño & 1990 \\
\hline
\end{tabular}

\title{
CAMBIOS GEOMORFOLOGICOS EN BARRANCOS POR EFECTO DE LAS REPOBLACIONES FORESTALES: EL CASO DE LOS RELIEVES DEGRADADOS DE LA DEPRESION NOROCCIDENTAL DEL EBRO
}

\author{
Luis M. ORTIGOSA*
}

\begin{abstract}
RESUMEN.-Se han seleccionado 8 pequeñas cuencas en relieves de la Depresión noroccidental del Ebro (La Rioja), de modo que la mitad de ellas tienen repoblaciones forestales $y$ las restantes sirven como testigos del estado ambiental previo. En un total de 29 barrancos se ha llevado a cabo un muestreo sistemático sobre el terreno para recoger información geomorfológica y de densidad de la cubierta vegetal de los lechos de inundación y los taludes limitantes. Después de analizar estadisticamente los datos, se concluye que el desarrollo forestal de las repoblaciones de coniferas influye positivamente sobre el ciclo del agua en las laderas y el funcionamiento hidromorfológico de los barrancos.
\end{abstract}

SUMMARY.-Geomorphological changes in gullies by the effect of afforestation: the case of the degraded areas of the Northwestern part of the Ebro Depression. Eight little basins in the northwestern part of the Ebro Depression (Spain, La Rioja) have been selected, in a way that half of them have afforestation and the rest of them serve as a proof of the previous envinronmental state. We choose 29 gullies, in wich has been made a sistematic samplig in the field in order to get geomorfological information and the density of the forest covert of the beds and the limitant talus. When analised statistically the data, we conclude that the coniferous afforestation development have a positive effect on the water cycle in the slopes and the hydromorfological behaviour of the gullies.

Palabras clave: Replobaciones, lechos fluviales, barrancos, erosión, Depresión del Ebro.

Key words: Reforestations, fluvial channels, gullies, erosion, Ebro Depression.

*Departamento de Geografia y Ordenación del Territorio. Colegio Universitario de La Rioja. 26001Logroño. 
Uno de los objetivos prioritarios de las repoblaciones forestales es el de la restauración hidromorfológica de laderas y cuencas, para conseguir de ese modo frenar la erosión de los suelos, aprovisionar de agua a las cuencas y evitar las frecuentes inundaciones. Esa es concretamente la finalidad perseguida en numerosos programas de repoblación relacionados con obras hidraúlicas, con los que se pretende reducir el rápido aterramiento de los embalses y alargar su vida útil, como asimismo en las repoblaciones de ambientes muy degradados y sometidos a procesos de erosión muy intensos (cabeceras fluviales activas, áreas abarrancadas, etc.). Como es bien sabido, los bosques controlan mejor que las formaciones de matorral o herbáceas el ciclo del agua en las laderas. Las cubiertas arbóreas interceptan la precipitación, retardando la caída de la lluvia y limitando su impacto sobre el suelo (splash). Además, los troncos y sistemas radiculares facilitan la infiltración y, por tanto, se reducen las tasas de escorrentia superficial (veáse, por ejemplo, LEE, 1980; LóPEZ CADENAS y BLANCO CRIADO, 1978; TOY, 1977).

Sin embargo, las propias tareas de reforestación llevan consigo una importante alteración medioambiental, que afecta negativamente a la estabilidad de los suelos y al comportamiento hidromorfológico de las vertientes. En primer lugar, se elimina una buena parte de la vegetación espontánea, especialmente matorrales de todo tipo, ya que son plantas muy competitivas para los nuevos árboles. Por otro lado, los métodos de plantación se basan en remover gran parte del material superficial de las laderas, tanto para enraizar los plantones como para crear estructuras microtopográficas de aprovisionamiento y regulación hídrica (microbanquetas, surcos, caballones, terrazas de bulldozer). Lógicamente, durante los primeros años de una repoblación, los pequeños árboles apenas ejercen ninguna función sobre la dinámica del agua en las vertientes y, por el contrario, el impacto repoblador puede favorecer la erosión de los suelos volteados y desprotegidos de vegetación. Diversos estudios han demostrado que las áreas repobladas presentan tasas de denudación especialmente elevadas, en muchas ocasiones con pérdidas irreversibles de los mejores horizontes del suelo utilizados para formar los caballones y terrazas (PAINTER et al, 1974; GARCIA-RUIZy ORTIGOSA, 1989; ORTIGOSA, 1990a). A su vez, los cauces, según las observaciones de BinNs (1979) en Gran Bretaña, muestran un incremento de los caudales y una reactivación erosiva de sus lechos, si bien a medida que crece el arbolado y se consiguen ambientes más estables y nemorales disminuye la escorrentia que alcanza los cauces y se reduce la exportación de sedimentos.

Siguiendo estos planteamientos, el objetivo del presente trabajo es comprobar los efectos hidrológicos que a medio plazo ejercen las repoblaciones forestales a escala de cuencas. A esta escala, el grado de actividad geomorfológica de los cauces representa un excelente medio de estudio. ya que sintetiza el comportamiento general de las vertientes. Como bien han explicado otros autores (SCHUMm, 1977; GARCIA-Ruiz et al, 1987), los balances de erosiónsedimentación e infiltración-escorrentía presentan puntualmente un comportamiento complejo a lo largo de las laderas, pero esta información se acumula globalmente en la red fluvial a través de la capacidad de transporte, los picos 
de crecida y la dinámica morfológica de sus lechos. Por ello, hemos centrado nuestro interés en analizar algunos aspectos geomórficos y ambientales de los cauces, tanto de cuencas repobladas como de cuencas testigo, siguiendo los métodos de campo empleados por GARCÍA-RuIZ y ORTIGOSA (1988). El estudio comparado de los cauces en áreas reforestadas y no reforestadas, nos permitirá sacar algunas conclusiones acerca del papel que juegan las plantaciones de conifferas en la restauración hidrológica de los ambientes degradados de la Depresión noroccidental del Ebro.

\section{Area de estudio}

La Depresión del Ebro, localizada en el tercio septentrional de la Península Ibérica, se caracteriza por una topografia de suaves llanuras, resultado de extensas rampas de glacis y distintos niveles de terrazas fluviales. Jalonando estas formas alomadas propias de las grandes cuencas de sedimentación terciaria y cuaternaria, encontramos también relieves más abruptos, residuos de la erosión de antiguos niveles de glacis o producidos por la incisión fluvial, que ponen al descubierto sus estructuras tabulares o subhorizontales y una litologia alternante con predominio de areniscas, arcillas, margas y yesos. En contraposición al extenso aprovechamiento agrícola de las llanuras, los relieves sobreelevados y las laderas escarpadas presentan en la actualidad un uso muy marginal, aunque en el pasado sufrieron la deforestación, el sobrepastoreo e incluso la puesta en cultivo mediante bancales. Estas actuaciones poco conservacionistas han favorecido la progresiva degradación de estos espacios, que por la pendiente de sus laderas son aún más susceptibles a la erosión de los suelos y al deterioro de su potencial biológico.

El sector más noroccidental de la Depresión, correspondiente en su mayor parte a la provincia de La Rioja, se desarrolla entre los 380 y $650 \mathrm{~m}$. de altitud. Sus relieves marginales se caracterizan además por una escasa elevación (50$200 \mathrm{~m}$. de desnivel sobre la base topográfica) pero con laderas de moderadafuerte pendiente y divisorias generalmente aplanadas. En ellas se inscriben pequeñas cuencas (del orden de decenas de hectáreas), con una red de drenaje muy activa, donde se pone de manifiesto la intensidad del escurrimiento superficial y la exportación de sedimentos de las laderas. Estas soportan suelos erosionados, poco potentes o incluso inexistentes. La vegetación más común es la formación dispersa de matorrales xéricos y camefiticos (tomillos, lavandas, etc.), acompañada de una cubierta de herbáceas más o menos abierta; la vegetación climácica de encinares y carrascales ha desaparecido en la mayoría de estos territorios, debido en gran parte al fuego reiterado para mejorar temporalmente el pastizal. Por su parte, el régimen termopluviométrico favorece la disgregación de los suelos y limita la regeneracióin vegetal, ya que el clima mediterráneo de matiz continental ( $11-12^{\circ}$ de temperatura media anual y 400 $500 \mathrm{~mm}$. de precipitación total) presenta un periodo estival de elevadas temperaturas y un acusado déficit hídrico, que se traduce en unos suelos muy 


\section{ORTIGOSA}

secos durante varios meses, afectado por precipitaciones esporádicas torrenciales. De hecho, muchos de estos espacios presentan características propias de las áreas acarcavadas.

En consecuencia, la deforestación histórica del paisaje explica el interés de la Administración por llevar a cabo a partir de los años 40 diversos proyectos de restauración forestal, con la finalidad de mejorar las condiciones ambientales y -en menor medida- para obtener a largo plazo un aprovechamiento forestal. Son manchas de repoblación de modestas dimensiones, realizadas con especies bien adaptadas a suelos pobres y a la sequía edáfica, como es el pino carrasco ( $P$. halepensis) y el ciprés (Cupressus sp.). Se han realizado con diversas técnicas, aunque para las más antiguas se emplearon métodos manuales (hoyos) o de tracción animal (fajas de buey). Entre estas últimas, hemos seleccionado precisamente para este trabajo cuatro cuencas repobladas hace 30 años aproximadamente, cuyos vuelos de copas cubren al menos en un 50$75 \%$ la superficie de suelo. Hemos considerado también otras tantas cuencas no reforestadas, localizadas en las proximidades y de características muy similares. Estas cuencas de estudio, emparejando una cuenca repoblada y otra testigo, se localizan en el Alto de La Grajera (Logroño), en el término de Arrubal y en los Agudos (Calahorra), donde hemos tomado dos conjuntos de cuencas (figura 1).

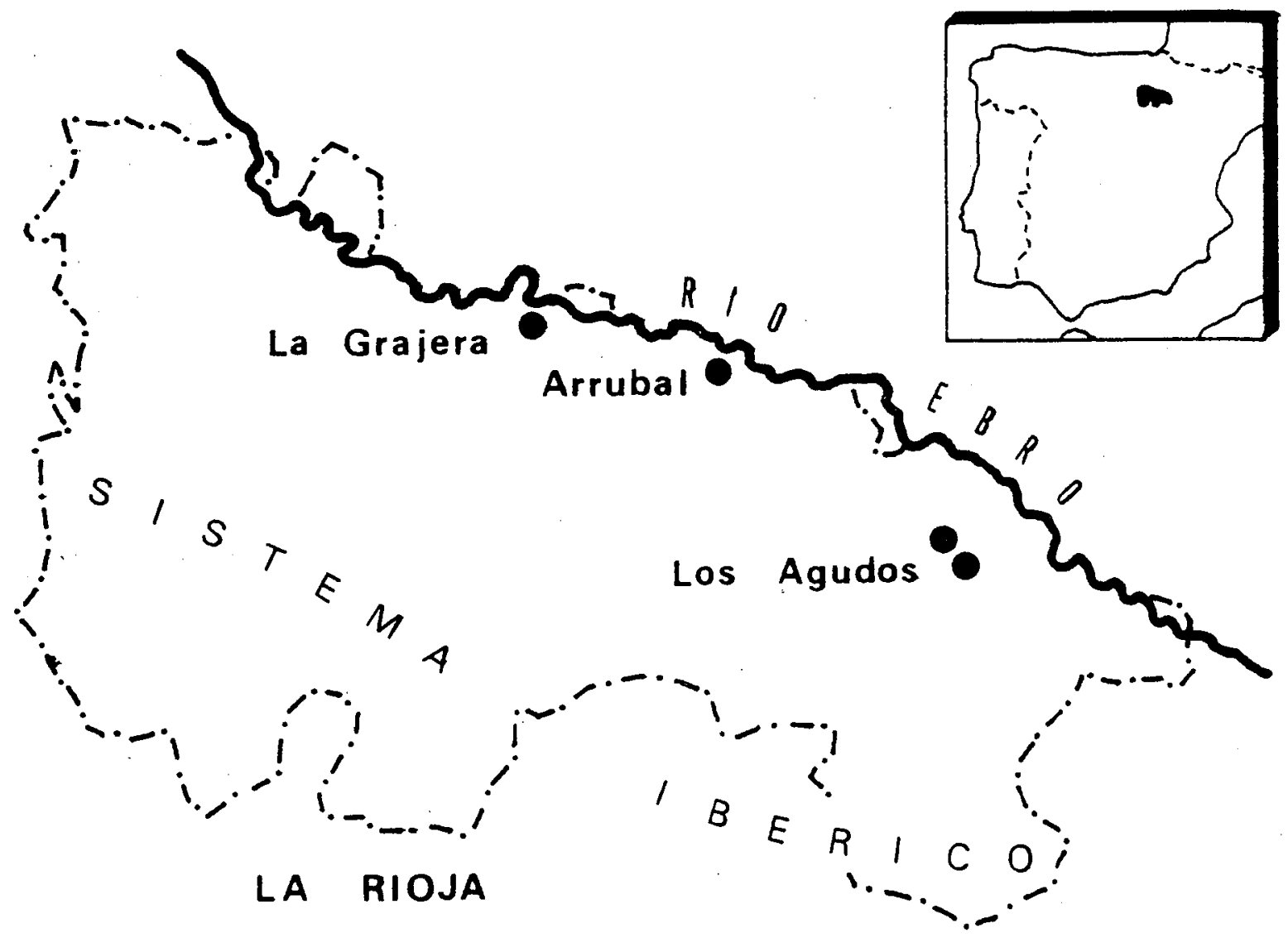

Fig. 1. Localización de las cuencas muestreadas. 
BARRANCOS Y REPOBLACIONES FORESTALES

\section{Métodos}

En cada una de las 8 cuencas seleccionadas se han muestreado 3 o 4 barrancos, procurando que fuesen cauces de distinto orden jerárquico. En total hemos trabajado sobre 29 tramos fluviales, recogiendo información de los lechos actuales y de los taludes limitantes; estos taludes enlazan las vertientes con la incisión de los barrancos, con pendientes muy pronunciadas pero de escasa altura (de uno a varios metros). En cada barranco se han tenido en cuenta 10 puntos de muestreo, distanciados entre sî 5 metros a lo largo de su trazado, en los que se anotó en un ficha de campo la siguiente información acerca del lecho fluvial: cubrimiento vegetal (\%) y suelo desnudo (\%) -ya que estos valores no tienen necesariamente que ser complementarios-, distinguiendo además la proporción de material fino, cantos o gravas y sustrato al descubierto, todo ello en porcentajes calculados a partir de jalones reglados. Por otro lado, en los taludes limitantes se calculó el porcentaje de cobertura vegetal, asi como la presencia relativa de procesos de erosión (\%), diferenciando cuatro microambientes geomorfológicos:

1. Canales e incisiones por arroyamiento concentrado.

2. Movimientos en masa: deslizamientos o pequeños slumps.

3. Areas de arroyamiento difuso fuerte, con suelos muy lavados y micropedestales.

4. Taludes estables con erosión moderada o nula, caracterizados por una densa cubierta vegetal protectora.

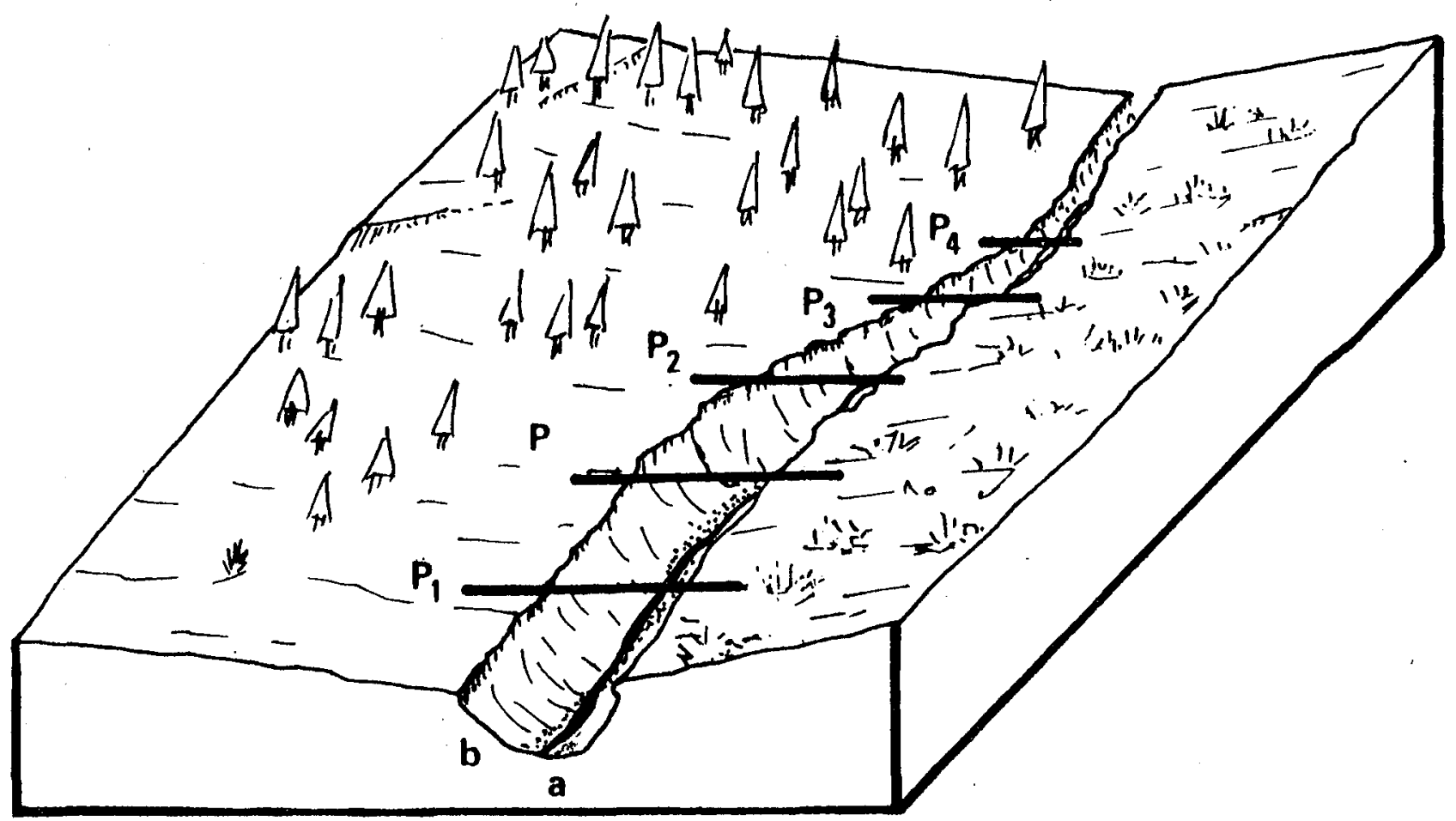

FIg. 2. Esquema representativo del muestreo sistemático realizado en 10 perflles transversales al barranco $\left(P_{n}\right)$, tomando diversa información de los lechos actuales (a) y de los taludes limitantes

(b). Metodologia tomada de Garcia-Ruiz y OrTigosa (1988). 
Posteriormente, todos estos datos de campo han sido procesados individualmente para cada barranco, calculando el valor medio porcentual de los parámetros cuantificados (cubrimiento vegetal de taludes y lechos, suelo desnudo, etc.). Finalmente, esta información más sintética se ha empleado para aplicar procedimientos estadísticos de cáracter bivariable (análisis de varianza y correlaciones lineales).

\section{Caracterización de la dinámica geomorfológica de los barrancos}

En general, los cauces estudiados son muy dinámicos, tal y como cabe esperar de unas vertientes con una débil protección vegetal, suelos poco potentes y arcillosos. La mayor parte son barrancos de vertiente (1-2 orden jerárquico), con caudales muy discontinuos pero con una notable potencia de arrastre y capacidad de erosión. Los lechos de inundación presentan una alta proporción de materiales finos (por término medio ocupan un $41 \%$ del lecho), hidricamente secos la mayor parte del año, pero escasamente colonizados por la vegetación, lo que demuestra la movilidad de estos sedimentos acumulados especialmente tras el receso de los picos de crecida o procedentes de los taludes laterales. En pequeños umbrales coincidentes con niveles más resistentes de areniscas y, sobre todo, en cauces de primer orden, aparece con frecuencia el sustrato rocoso, resultado del transporte e incisión de estos canales. Asimismo, los taludes presentan evidencias de una constante evolución geomorfológica, especialmente por la reactivación impuesta por la socavación lateral del canal (zapamiento de la base del talud), incentivando así los desprendimientos por tensión gravitacional. El cubrimiento vegetal de los taludes es relativamente escaso ( $44 \%$ en promedio) debido a su inestabilidad por diferentes mecanismos de erosión: movimientos en masa, pequeños regatos o intenso arroyamiento difuso. No es dificil observar en ellos la deposición de tepés, paquetes de matorral o incluso pinos de repoblación, desprendidos por el retroceso de la parte superior del talud. Sobre todo al pie de las vertientes (cauces de tercer orden), los canales presentan fondo plano y paredes muy verticales, resultado de los procesos de piping o sufosión (GUTIÉRREZ-ELORZA y RoDRÍGUEz-VIDAL, 1984).

No obstante, el grado de actividad de estos barrancos varia sensiblemente de unos a otros, como podemos comprobar a partir de los datos de la tabla 1. Algunos cauces presentan una morfogénesis muy dinámica (escasa c0olonización vegetal, gran proporción de sustrato al descubierto, taludes muy inestables), mientras que otros tienen un comportamiento más ponderado y menos cambiante. En cualquier caso, podemos comprobar la relación existente entre el funcionamiento de los lechos fluviales y sus respectivos taludes, de manera que los barrancos con taludes desnudos y una alta proporción de procesos erosivos coinciden de modo general con lechos desprotegidos de vegetación, sustratos 
exhumados y alta proporción de cantos. Como ejemplo de ello, en la figura 3 podemos observar la relación entre el cubrimiento vegetal de cauces y el de los taludes $(r=+0,647)$.

\section{TABLA 1}

Caracteristicas descriptivas de los cauces y taludes muestreados.

\begin{tabular}{|c|c|c|c|c|}
\hline CAUCES & Promedio & Desu. estándar & Máximo & Minimo \\
\hline Cub. vegetación(\%) & 47,3 & 21,1 & 86 & 8 \\
\hline $\begin{array}{l}\text { Suelo desnudo(\%) } \\
\text { composición: }\end{array}$ & 58,9 & 25,2 & 96 & 17 \\
\hline Finos(\%) & 41,0 & 19,4 & 75 & 5 \\
\hline Cantos(\%) & 14,2 & 10,3 & 46 & 0 \\
\hline Sustrato(\%) & 3,6 & 5,8 & 21 & 0 \\
\hline TALUDES & Promedio & Desv. estándar & Máximo & Mínimo \\
\hline Cub. vegetación(\%) & 43,8 & 13,1 & 63 & 12 \\
\hline \multicolumn{5}{|c|}{ Microambientes geomorfológicos: } \\
\hline Eros. moderada(\%) & 40,5 & 15,2 & 67 & 7 \\
\hline Canales(\%) & 8,1 & 4,9 & 20 & 0 \\
\hline Movimientos en masa(\%) & 23,5 & 11,3 & 46 & 2 \\
\hline E.difusa fuerte(\%) & 27,6 & 6,4 & 37 & 13 \\
\hline
\end{tabular}

Para comprender la diversidad morfogenética de estos barrancos, hay que considerar dos importantes factores. Primeramente, la heterogeneidad de las áreas estudiadas, ya que las cuencas -testigos y repobladas- de La Grajera o Arrubal tienen un relieve más moderado y unas condiciones ambientales menos degradadas que las de Los Agudos, que presentan barrancos muchos más activos. En segundo lugar, hay que tener en cuenta la jerarquía de los barrancos. La mitad de ellos correponden a cauces de primer orden, sin afluentes, inscritos en sectores topográficos pronunciados y generalmente con un gradiente longitudinal elevado. Estos cauces, en comparación con otros más integrados en la red o desarrollados en la base de las cuencas, demuestran una mayor capacidad incisiva y -en algunos casos, como veremos más adelante- una activa erosión remontante. Al margen de estos aspectos diferenciadores, la repoblación forestal es también un factor decisivo para explicar un comportamiento más estable y menos erosivo de los barrancos que drenan estas cuencas. 


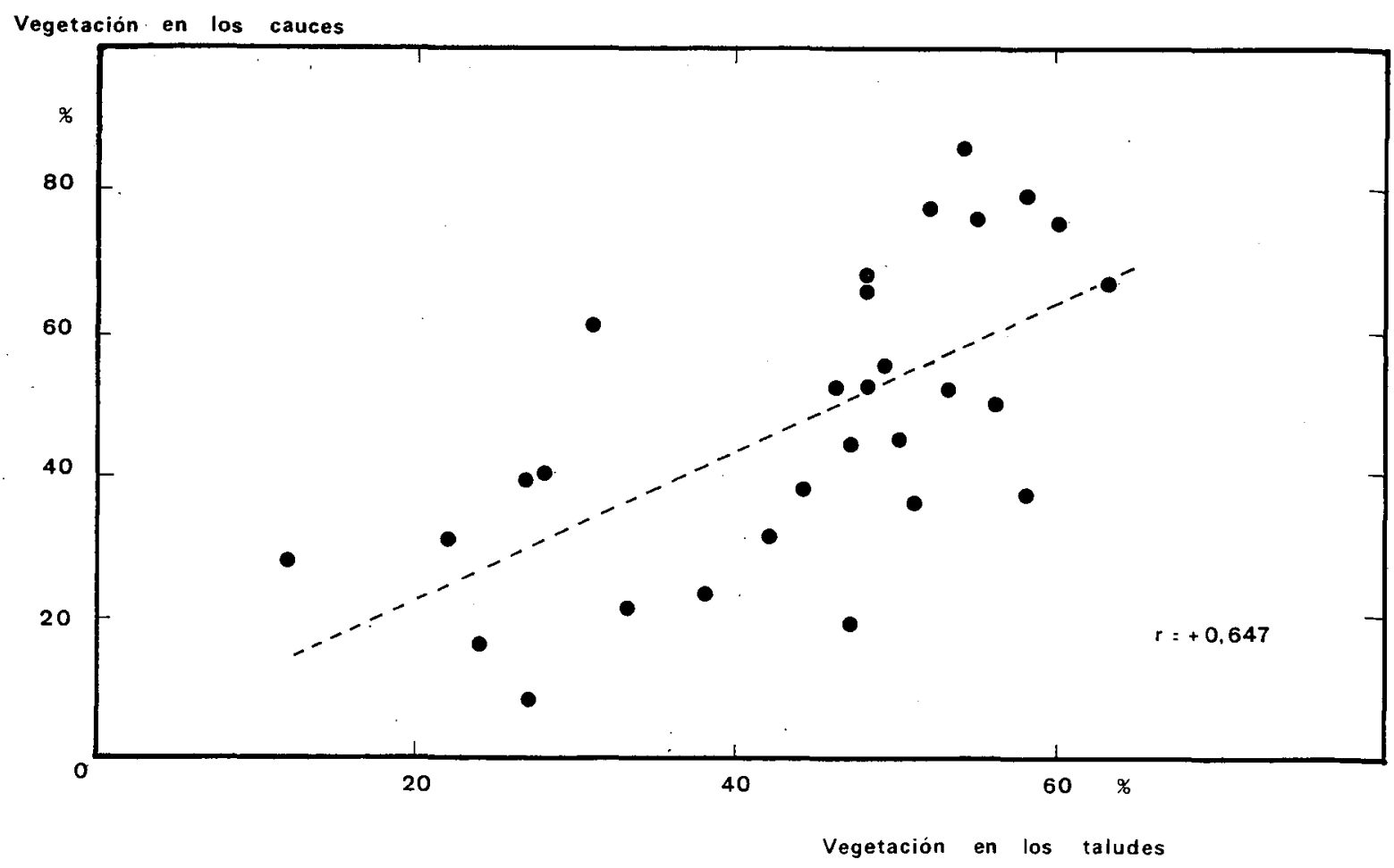

Fig. 3. Relación entre el cubrimiento de la vegetación en los lechos de inundación y en los taludes de los barrancos.

\section{Efectos de la repoblación forestal en los cauces}

Al evaluar mediante análisis de varianza las características de los barrancos según correspondan a cuencas repobladas o cuencas testigo, se aprecian algunas diferencias significativas desde el punto de vista estadístico (veáse la tabla 2). Los lechos actuales de cauces en vertientes reforestadas presentan por término medio una mayor cobertura de vegetación (53,9\%) frente a los de las cuencas no repobladas $(41,2 \%)$. Estos últimos denotan suelos más desnudos y transportan mayor cantidad de material grueso $(17,5 \%)$ que los canales de áreas con repoblaciones $(10,6 \%)$. El sustrato apenas es visible en los barrancos de las vertientes repobladas $(0,9 \%)$, pero es mucho más frecuente en los de áreas testigo $(6,1 \%)$, por lo que debemos pensar que su potencia hidraúlica es también mucho más acusada. De modo similar, los escarpes adyacentes a los cauces son más estables en los perímetros repoblados, mientras que los de cuencas testigo presentan una mayor erosión (erosión moderada o nula en los taludes: 45,6 y $35,8 \%$, respectivamente). Aunque las diferencias son reducidas, parece ser que los desprendimientos y el arroyamiento superficial son menos frecuentes en los taludes de barrancos correspondientes a repoblaciones.

Estos efectos estabilizantes de las repoblaciones forestales en la dinámica geomorfológica y sedimentaria de los cauces se observa en todos los conjuntos de cuencas repobladas-testigo. Pero los datos indican que las diferencias más 
acusadas se aprecian en aquellas vertienes que partian de unas condicioens ambientales más regresivas (Los Agudos y relieves de Arrubal). En las cuencas de La Grajera, con formaciones vegetales más cerradas y suelos menos degradados, la influencia de la reforestación sobre lechos y taludes es menos contrastada. Por otra parte, la cubierta forestal de la cuenca no ejerce el mismo control geomorfológico sobre todo tipo de cauces. Los barrancos de primer orden correspondientes a áreas repobladas, aunque ofrecen también un aspecto muy activo de sus lechos y taludes, apenas presentan la roca subyacente por la incisión de los canales. En los de vertientes sin arbolado, estos barrancos transportan fácilmente la carga de fondo y aún mantienen energía para descubrir la roca subyacente; éste es el aspecto más diferenciador entre los barrancos de primer orden de cuencas repobladas y de cuencas testigo (ver la figura 4A). Los canales de segundo y tercer orden, tanto en áreas forestadas como no repobladas, no tienen una actividad incisiva tan dinámica (aparece muy poco el sustrato) y los taludes son relativamente más estables; el contraste más evidente entre unos y otros barrancos está en relación precisamente con la frecuencia de desprendimientos en los taludes, notablemente más activos en los cauces de áreas no repobladas (figura 4B).

TABLA 2

Valores medios de las caracteristicas de los cauces en cuencas repobladas y cuentas testigos

\begin{tabular}{lccc}
\hline CAUCES & $\begin{array}{c}\text { Cuencas } \\
\text { repobladas }\end{array}$ & $\begin{array}{c}\text { Cuencas } \\
\text { testigos }\end{array}$ & $\begin{array}{c}\text { Significación } \\
\text { ANOVA }\end{array}$ \\
\hline Cub. vegetación(\%) & 53,9 & 41,2 & $0,036^{*}$ \\
Suelo desnudo(\%) & 48,9 & 67,9 & \\
composición: & & & \\
& 10,6 & 17,5 & $0,035^{*}$ \\
Cantos (\%) & 37,4 & 44,3 & 0,217 \\
Mat.fino(\%) & 0,9 & 6,1 & $0,011^{*}$ \\
Sustrato(\%) & & & \\
& Cuencas & Cuencas & Significación \\
& repobladas & testigos & ANOVA \\
\hline TALUDES & 45,6 & 35,8 & $0,051^{*}$ \\
microambientes: & 5,4 & 10,6 & $0,002^{*}$ \\
\hline Erosión moderada(\%) & 23,4 & 24,5 & 0,576 \\
Canales(\%) & 26,6 & 28,9 & 0,293 \\
Mov. en masa(\%) & & & \\
E. difusa fuerte & & & \\
\hline
\end{tabular}

Estos hechos debemos explicarlos porque el desarrollo del arbolado de repoblación ejerce efectivamente una influencia positiva en el funcionamiento hidrológico de las vertientes, al menos en cuencas que tenian el momento anterior a la repoblación un balance infiltración-escorrentía muy negativo; en esas áreas, en virtud del desarrollo de una densa cubierta de coniferas, se frena 


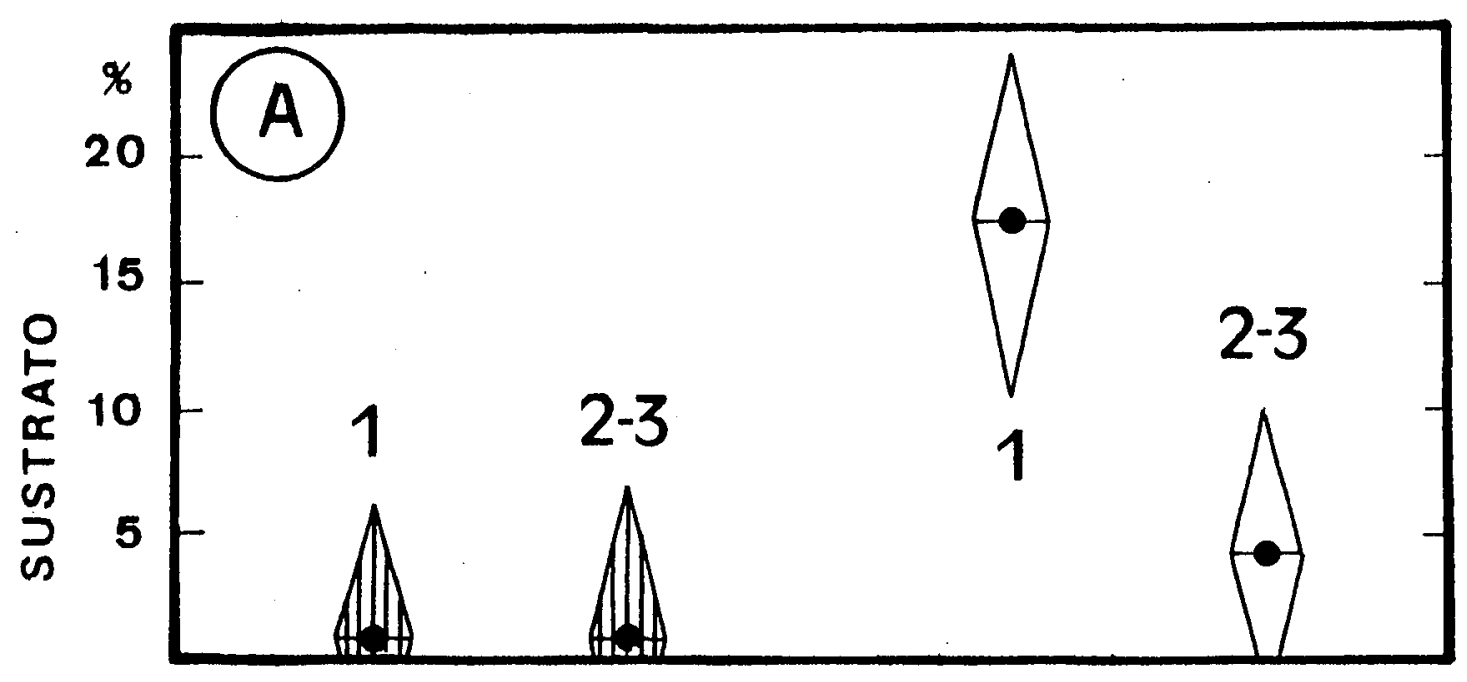

Cuencas:

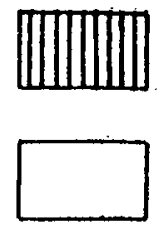

Repobladas

Testigos
Orden de los cauces:

1,2 o 3

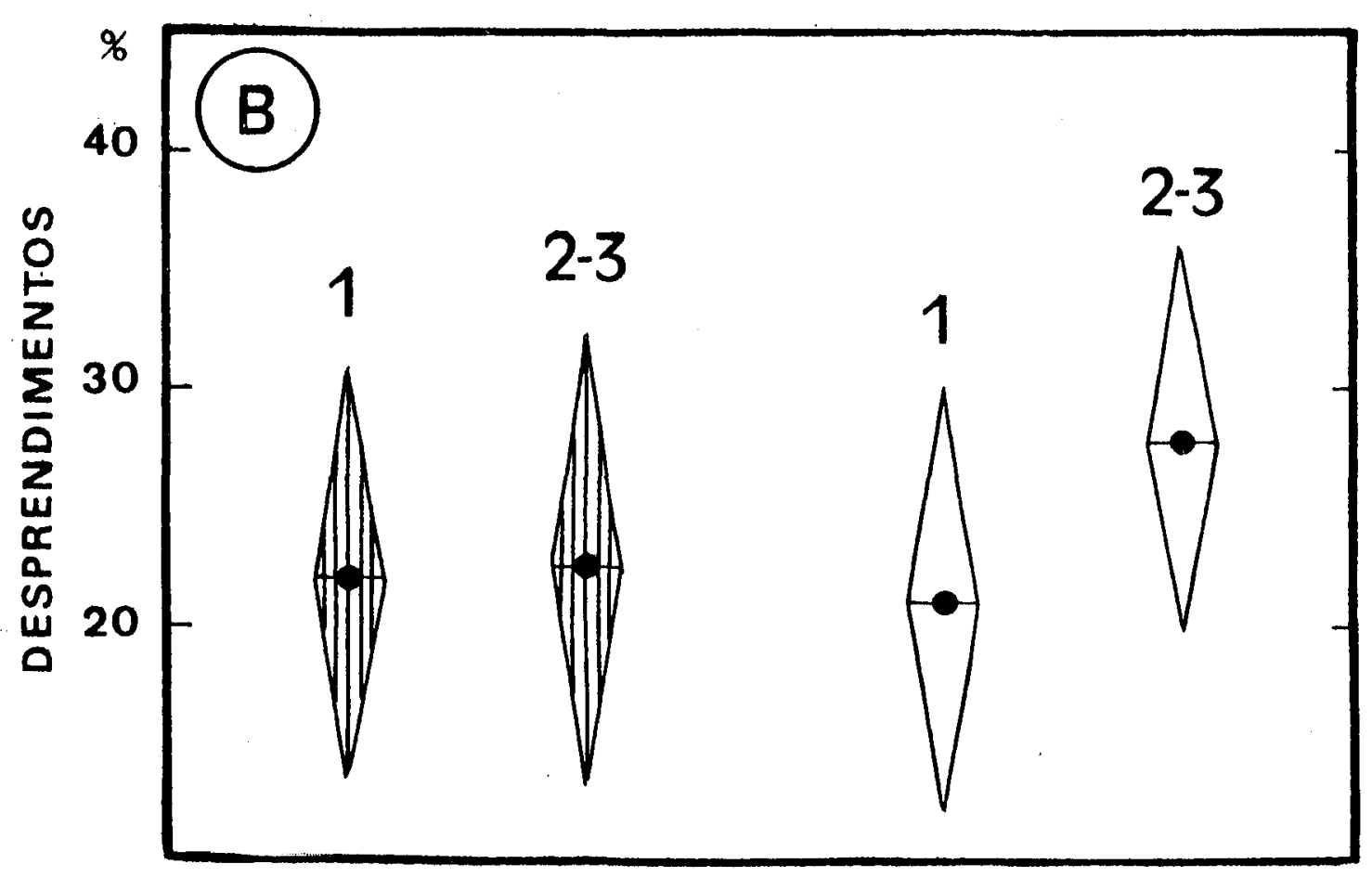

Fig. 4. Valores medios y dispersión (desvlación estándar) de (A) la presencla de sustrato en los canales y (B) la frecuencla de desprendimlentos en los taludes, distingulendo el orden de los cauces y el tipo de cuencas. 
en alguna medida la escorrentia instantánea que alcanza los cauces, retardando su drenaje y haciendo disminuir los picos de crecida. Los barrancos de primer orden atenúan la capacidad de exportación de sedimentos y su dinámica expansiva, por lo que en ellos apenas observaremos los niveles rocosos por incisión o remonte. En los cauces más importantes, que drenan mayores superficies de la cuenca y reciben a los pequeños arroyos, la concentración de caudales también será inferior, de modo que sus canales no sufren tanto el zapamiento de los bancos del lecho ni los desprendimientos de los escarpes limitantes. Hay que insistir en que los impactos producidos por el laboreo de la reforestación transforma negativamente el funcionamiento hídrico y erosivo de las vertientes y cauces, seguramente con una reactivación de los caudales y de las tasas de erosión con respecto a las áreas no repobladas. Pero pasada la fase inicial, a medida que adquieren importancia las estructuras forestales y se recolonizan los suelos removidos, la cuenca absorbe mejor los volúmenes de precipitación, que en último término implica una disminución de la arroyada superficial hacia los barrancos y una restauración hidrológica y geomorfológica.

\section{Conclusiones}

En los relieves destacados de la Depresión del Ebro, marginados para el uso humano pero caracterizados por un avanzado estado de degradación ambiental y una dinámica hidromorfológica muy exportadora, las repoblaciones forestales ejercen a medio plazo un control positivo sobre el ciclo del agua en las laderas y sus barrancos. Como se ha observado también en áreas repobladas del Pirineo Central (GARCíA-RuIZ y ORTIGOSA, 1988), los lechos y taludes de los cauces presentan un aspecto geomorfológico menos activo y una mayor estabilidad de las formas: mayor cubrimiento de la vegetación en los canales de inundación y en sus escarpes próximos, disminución del tamaño medio de materiales transportados, menor presencia del sustrato, etc. Estos efectos de las repoblaciones han sido también observados por diversos investigadores, aunque algunos reconocen que su acción es limitada e insuficiente como para detener las consecuencias de grandes precipitaciones (ClARKE y McCULLOCH, 1979; Gilmour et al, 1987; DOUGUEDRoIT, 1981).

Es evidente, pues, que las repoblaciones forestales implican cambios importantes en los mecanismos de transferencia de sedimentos y en las formas de circulación del agua en las laderas. Pero esta afirmación debería ser válida sólo cuando los resultados -en forma de densidad de la cubierta forestal- sean muy positivos. En este trabajo hemos considerado solamente perimetros con crecimientos aceptables para las condiciones ambientales de partida, de manera que muestran una cubierta vegetal significativamente diferente de la de las llamadas cuencas testigos. Por otra parte, las repoblaciones seleccionadas se han realizado con técnicas moderadamente agresivas, excluyendo los aterrazados de bulldozer, que tienden a aumentar la movilidad del suelo (ORTIGOSA, 1990b). Indudablemente, los resultados obtenidos en este trabajo serian muy diferentes 
ORTIGOSA

si se hubieran considerado repoblaciones con crecimientos muy pobres y con un elevado número de marras. En todo caso, se demuestra una vez más la eficacia de determinadas actuaciones sobre la cubierta vegetal para controlar la dinámica hidrológica y geomorfológica de vertientes y cauces, a condición de que los saltos en la sucesión vegetal no sean espectaculares (OrTIGOSA, 1990a) ni se altere profundamente el suelo mediante técnicas duras de repoblación.

\section{Bibliografia}

BINNS, W.O., 1979.- The hydrological impact of afforestation in Great Britain. Man's impact of hidrological cycle in the United Kingdom. (G.E. Holis, edr.), Geo-Books, 55-69 Pp. Norwich.

CLARKE,R.T. and MCCULLOCH,J.S.G., 1979.- The effect of land use on the hydrology of upland catchments. Man's impact of the hydrological cycle in the United Kingdom. (G.E. Holis, edr.), Geo-Books, 71-78 pp., Norwich.

DougueDRorT, A., 1981.- Reafforestation in the French Southern Alps. Mountain Research and Development, 1 (3-4): 245-252 pp.

GARCÍA-RUIZ,J.M., GómEZ-VILLAR,A. y OrTiGOSA,L.M., 1987.- Aspectos dinámicos de un cauce fluvial en el contexto de su cuenca: el ejemplo del rio Oja. Instituto de Estudios Riojanos e Instituto Pirenaico de Ecologia, 112 pp.

GaRcta-RUIZ, J.M., OrTiGosa, L.M., 1988.- Algunos efectos geomorfológicos de las repoblaciones forestales: cambios en la dinámica de cauces en pequeñas cuencas del Pirineo central español. Cuaternario y Geomorfología, 2 (1-4): 33-41 pp.

GaRCíA-Ruiz, J.M., ORTIGOSA, L.M. y MARTINEZ-CASTROVIEJo, R., 1988.- Nota sobre la geomorfologia de ambientes degradados del Pirineo aragonés. Homenaje a Pedro Monserrat, 983-991 pp., Instituto de Estudios Altoaragoneses e Instituto Pirenaico de Ecologia, Jaca.

GARCIA-RUIZ, J.M. y ORTiGOSA, L.M., 1989.- Efectos geomorfológicos de la repoblación forestal. En: Evolución ecológica de las areas reforestadas en el Pirtneo aragonés. Instituto Pirenaico de Ecologia y Estación Agricola Experimental de Aula Dei (CSIC), Jaca y Zaragoza.

Gilmour, D.A., BonEll, M. and CASSELS, D.S., 1987.- The effects of afforestation on soil hydraulic properties in the middle hills of Nepal. Mountain Research and Development, 7(3): 239-249 pp.

GuTtÉRREZ-Elorza, M. y Rodríguez-VidAL, J., 1984.- Fenómenos de sufosión (piping) en la Depresión media del Ebro. Cuadernos de Investigación Geográfica 10 (1-2): 7-15 pp., Logroño.

LEE, R., 1980.- Forest hydrology. Columbia University Press, 349 pp., New York.

López-CADEnas, F. y BlanCo-Criado, M., 1978.- Hidrología forestal. Escuela Técnica Superior de Ingenieros de Montes, 2 vol., Madrid.

MuRGatroYd, A.L. and TERNAN, J.L., 1983.- The impact of afforestation on stream bank erosion and channel form. Earth Surface Processes, 8: 357-369 pp.

ORTIGOSA, L.M., 1990a.- Las repoblaciones forestales como estrategia de intervención en regiones degradadas de montaña. En: Geoecología de las areas de montaña. (J.M. Garcia-Ruiz, edrd, Geoforma Ediciones, 297-311 pp., Logroño.

ORTIGOSA, L.M., 1990b.- Resultados y consecuencias ambientales de las repoblaciones forestales (Sistema Ibérico y Depresión noroccidental del Ebro). Geoforma Ediciones, Logroño (en prensa). 


\section{BARRANCOS Y REPOBLACIONES FORESTALES}

PAinTER, R.B., BLYTH, K., Mosedale, J.C. and Kelly, M., 1974.- The effect of afforestation on erosion processes and sediment yield. Effects of man on the interface of the hydrological cycle with the physical environment. International Association of Hydrological Sciences Publication, 113: 62-68 pp.

Schuмm, S.A., 1977.- The fluvial system. John Wiley, 338 pp., New York.

ToY,J.T. (edr.), 1977.- Erosion Research techniques, erodibility and sediment delivery . GeoBooks, 86 pp., Norwich. 
